\title{
An Experimental Study on Relationship Between Intellectual Concentration and Personal Mental Characteristics
}

\section{$\operatorname{AUTHOR}(\mathrm{S}):$}

Takekawa, Wakako; Ueda, Kimi; Ogata, Shogo;

Shimoda, Hiroshi; Ishii, Hirotake; Obayashi, Fumiaki

\section{CITATION:}

Takekawa, Wakako ...[et al]. An Experimental Study on Relationship Between Intellectual Concentration and Personal Mental Characteristics. Human Systems Engineering and Design 2018, 876: 560-566

\section{ISSUE DATE:}

2018

URL:

http://hdl.handle.net/2433/259835

\section{RIGHT:}

This is a pre-print of an article published in Human Systems Engineering and Design. The final authenticated version is available online at: https://doi.org/10.1007/978-3-030-02053-8 85.; この論文は出版社版でありません。引用の際には 出版社版をご確認ざ利用ください。; This is not the published version. Please cite only the published version. 


\title{
An Experimental Study on Relationship between Intellectual Concentration and Personal Mental Characteristics
}

\author{
Wakako Takekawa', Kimi Ueda', Shogo Ogata' ${ }^{1}$, Hiroshi Shimoda ${ }^{1}$, \\ Hirotake Ishii and Fumiaki Obayashi ${ }^{2}$ \\ ${ }^{1}$ Graduate School of Energy Science, Kyoto University, Kyoto, Kyoto, 606-8501, Japan \\ \{takekawa, ueda, ogata, shimoda, hirotake\}@ei.energy.kyoto-u.ac.jp \\ ${ }^{2}$ Panasonic Corporation, Kadoma, Osaka, 571-8686, Japan \\ obayashi.fumiaki@jp.panasonic.com
}

\begin{abstract}
As a proposal of new diagnosis for mental diseases, this study focused on the relationship between intellectual concentration and personal mental characteristics. It is expected that the measurement of concentration characteristics may help the diagnosis of the mental disorders because the mental characteristics such as psychiatric disease, developmental disorder and behavioral feature are supposed to be closely related to their mental activity such as concentration. When analyzing the relationship, the characteristics of concentration are expressed as 36 feature values by analyzing answering time distribution of cognitive task, and the values of concentration were compressed to 5 main factors by principal component analysis. Then the combination of the factors and one of 36 parameters of mental characteristics were given to a decision tree analysis tool.
\end{abstract}

Keywords: Intellectual concentration $\cdot$ Mental characteristics $\cdot$ Decision tree analysis

\section{Introduction}

In recent years, the number of patients with mental illness tends to increase [1]. In addition, it is difficult to establish reliable criteria for the diagnosis of these diseases, and this difficulty is seen not only for mental disorders but also for developmental disorders and so on [2]. In this situation, it is desired to realize more reliable diagnosis which can provide proper treatment to potential patients.

In this study, therefore, the authors focus on intellectual concentration and examine the possibility that it is closely related to their mental characteristics and its availability to one of the criteria for diagnosis of mental disorders. In addition, the relationships between the intellectual concentration and developmental disorders trend / personal characteristics are also investigates as one of the factors reflecting mental condition.

Regarding quantitative evaluation of intellectual concentration, various studies have been conducted. One of them define that the intellectual concentration can be 
expressed as a state transition model as shown in Fig. 1 [3]. The model consisits of "Concentration state" and "Non-concentration state" [4]. In the concentration state, the cognitive resources are assigned to the target task, while they take rest for a while in the non-concentration state. The concentration state is also divided into two states which are working state and short-term pause state. Based on this concept, indices indicating the ratio of concentration time (Concentration Time Ratio; CTR) [5] and the depth of concentration (Concentration Depth Index; CDI) [6] were developed.

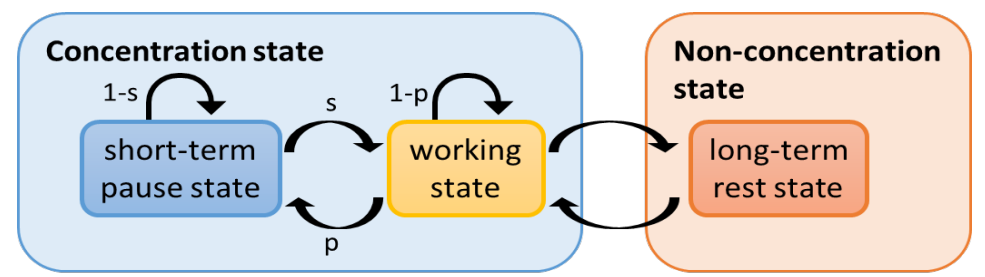

Fig. 1. Three state concentration model.

The purpose of this study is to conduct an experiment to gather the quantitative data of intellectual concentration and mental characterristics which are not only personal characteritics but also mental disorders and developmental disorders trend. And then the relationships between them are examined based on the collected data by statistical analysis.

\section{Method}

In this study, both personal mental characteristics and answering time data of cognitive tasks which showed the charactersitics of intellectual concentration were collected first, and then statistical analysis to find associations between the collected data was performed. This chapter describes the procedure of the experiment, the approximation of the concentration state using the answering time series of the cognitive task, and the outline of the statistical analysis.

\subsection{Experimental Procedure}

In this study, 236 university students of Kyoto University were recruited to join the experiment where their data of personal characteristics and answering time series of cognitive tasks were obtained. The participants received e-mails describing URLs to questionnaire survey pages about one week before the participation date of the experiment and they answered all the questionnaires via the internet in advance. The questionnaires will be explained in Section 2.2.

The protocol of the experiment is shown in Fig.2. "Comparison Task" developed by Ueda et al. was employed as the cognitive task in the experiment. It consists of problems of uniform difficulty requiring cognitive abilities to be used in office work [7].

The data of 10 participants were omitted because they slept during the task and those of 226 participants were analyzed in total. 


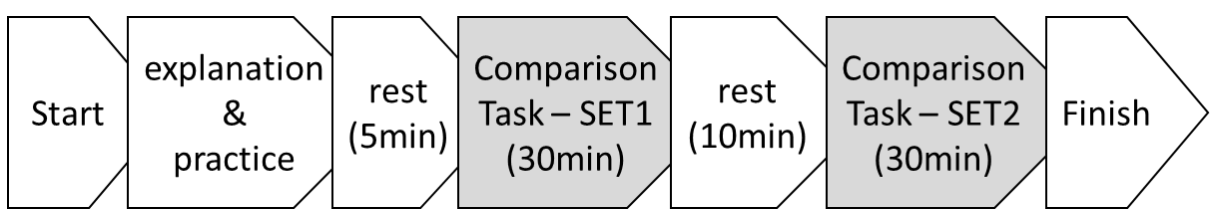

Fig. 2. Protocol of the experiment.

\subsection{Survey on Personal Mental Characteristics}

To investigate mental symptoms and personal characteristics, the following 6 surveys were conducted;

(1) "BIS/BAS scale" that shows the tendency of behavioral inhibition and behavioral approach [8],

(2) "Autism-spectrum Quotient (AQ)" that shows the degree of autism spectrum [9],

(3) "Yatabe-Guilford Personality Inventory (YG)" that finely extracts personal characteristics [10],

(4) "General Health Questionnaire (GHQ)" that shows whether to have tendency of neurosis [11],

(5) "Global Scale for Depression (GSD)" that shows the presence and the type of depression [12] and

(6) "NEET/Hikikomori Risk Scale" that investigates psychological tendencies common to those having problems in social adjustment [13].

\subsection{Quantitative Evaluation of Intellectual Concentration}

Fig. 3 shows an example of the answering time histogram of the cognitive task.

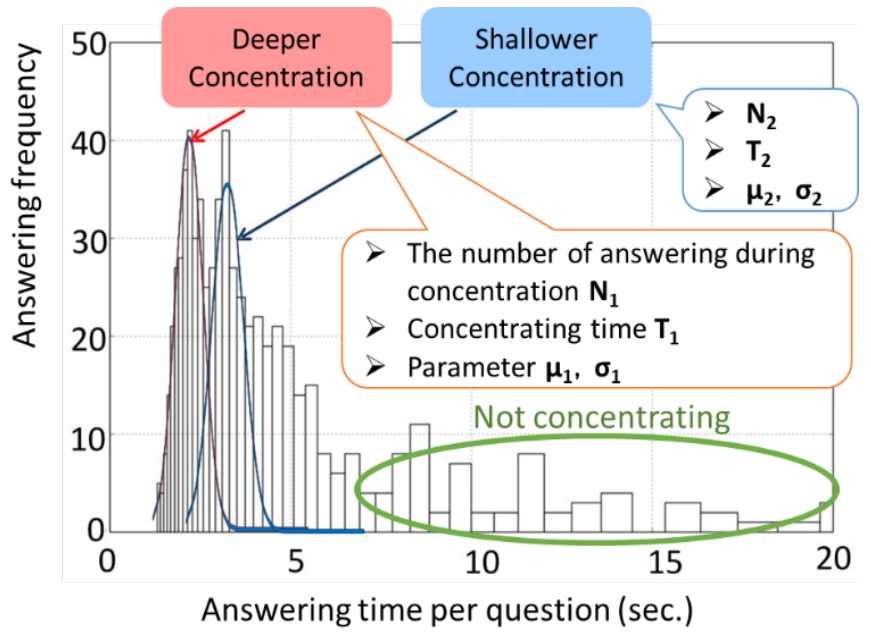

Fig. 3. Histogram of answering time. 
The distribution can be divided into concentration and non-concentration state, and then the former can be approximated by sum of two lognormal distributions; deeper concentration and shallower concentration, based on the concept of three state model [4] described in Section 2.1. In this approximation, the number of answers in the concentration state, the duration of concentration, the parameters of the lognormal distribution, and so on were calculated as feature values which expressed the intellectual concentration as shown in Table 1.

Table 1. Feature values to express intellectual concentration.

\begin{tabular}{|c|l|}
\hline Feature value & Meaning \\
\hline $\mathbf{N}$ & The number of total answering \\
\hline $\mathbf{N}_{1}$ & The number of answering while deeper concentration \\
\hline $\mathrm{N}_{2}$ & The number of answering while shallower concentration \\
\hline $\mathrm{T}_{1}$ & The time of deeper concentration \\
\hline $\mathrm{T}_{2}$ & The time of shallower concentration \\
\hline $\mathrm{CTR}^{*}$ & Concentration time ratio \\
\hline $\mathrm{MCTR}$ & Multi concentration time ratio \\
\hline $\mathrm{CDI}^{*}$ & Concentration depth index \\
\hline$\mu_{1}^{*}, \sigma_{1}^{*}$ & Paratemer of log-normal distribution showing deeper concentration \\
\hline$\mu_{2}^{*}, \sigma_{2}^{*}$ & Paratemer of log-normal distribution showing shallower concentration \\
\hline
\end{tabular}

In addition, some values were added to express the detail features of the intellectual concentration. Fisrt, the ratio of $\mu_{2} / \mu_{1}$ and $\sigma_{2} / \sigma_{1}$ were added since it could express the difference between deeper and shallower concentration. Then, for the values of $*$ mark in Table $1, \mu_{/} / \mu_{1}$ and $\sigma_{2} / \sigma_{1}$, the ratio between the value at SET1 and SET2 was added since it could express the changes in intellectual concentration and could be quantified when resuming work before and after a short break.

In this way, total 36 feature values were used as explanatory variables in the later analysis.

\subsection{Decision Tree Analysis}

As an analytical method to investigate the relationship, decision tree analysis was used in this study. The greatest advantage of this method is that it is easy to visually judge the elements which are important or not for the conclusion, and the result can be easily interpreted.

The results of personal mental characteristics survey were used for the objective variable. Regarding AQ, GHQ and GSD, the presence or absence of symptoms judged from the score was set as objective variables, and regarding BISBAS, YG and NEET/Hikikomori, scores themselves were set.

On the other hand, the indices of intellectual concentration were set as explanatory variable. They were standardized and then compressed into five indices by principal component analysis, because the number of total feature values, 36, is too numerous and effective results can not be obtained.

The contents indicated by the five principal components were (1) answering time, (2) deeper concentration, (3) improvement in the work speed or concentration depth 
after the break, (4) shallower concentration, (5) decrease in the concentration after the break.

The procedure of the above analysis is shown in Fig.4. "Scikit-learn" was used for the analysis, which is open source machine learning library in Python developed by Pedregosa et al.[14].

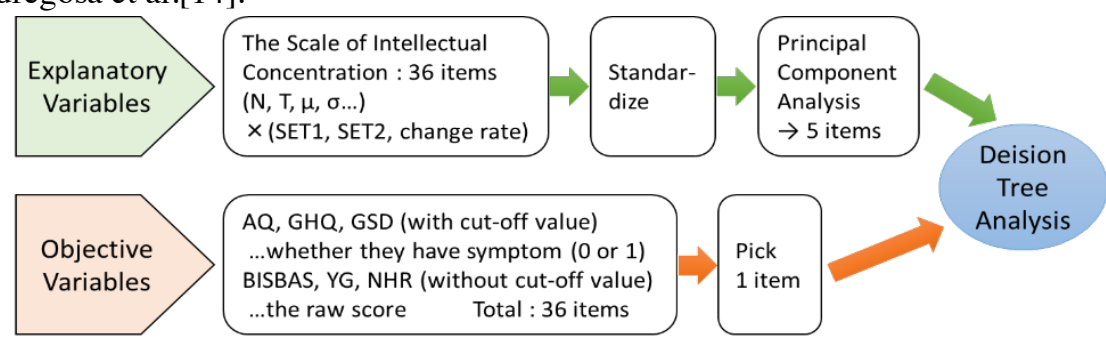

Fig. 4. Procedure of analvsis.

\section{$3 \quad$ Result \& Discussion}

The analysis result was expressed as an inverted tree-like figure. Fig. 5 shows an example of the analysis result where AQ was set as an objective variable.

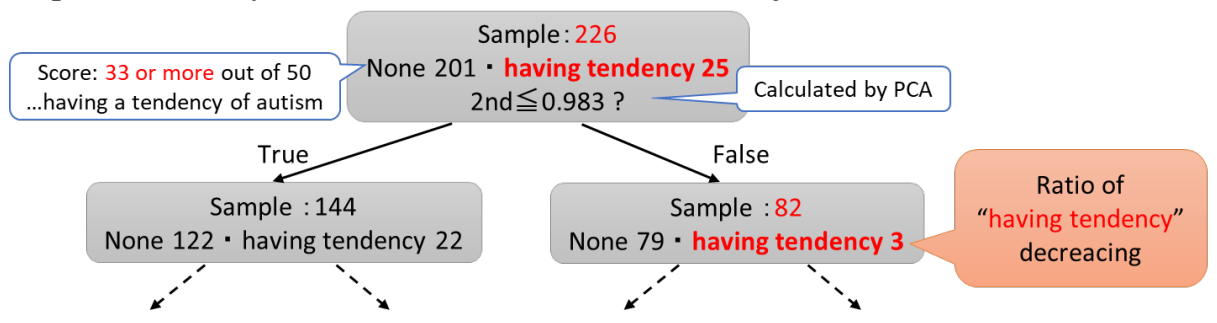

Fig. 5. Simplified figure of the result - AQ.

In this example, the branching condition is True when the 2nd principal component is 0.983 or less, and it is False when it is larger than 0.983 . The 2nd principal component is the component indicating the degree of deeper concentration as described in Section 2.3, so 82 perticipants that branched to the False side have relatively large degree of deeper concentration. Then focusing on the proportion with autistic tendency, $25(11.1 \%)$ of 226 perticipants have the tendency in total, while only 3 $(3.7 \%)$ out of 82 who branched to False side. In other words, it is supposed that a person with a relatively deeper concentration is likely not to have the autistic tendency.

Other tendencies were also found as the results of the analysis as shown below; (a) People with relatively high concentration are likely to be obedient. (b) People with relatively low concentration are likely to have a temperament like job-hopping parttimers. (c) People who get slow in work speed after a break are likely to be emotional. (d) People who improve their concentration after a break are likely to be confident, outgoing and active. On the other hand, no notable relationship was found regarding with GHQ and GSD. From the above analysis, some personal mental characteristics which were likely to be related to intellectual concentration were extracted. 


\section{Conslusions}

In this study, an experiment to collect quantitative data of intellectual concentration and personal mental characterristics was conducted. And then the relationships between them were examined based on the collected data using statistical methods. As the results, it was found that some mental characteristics were related to the intellectual concentration. At the current stage, however, it is difficult to practically use the indices of intellectual concentration as one of the diagnostic basis for symptons or criteria for personal characteristics.

Acknowledgments. This work was supported by JSPS KAKENHI Grant Number JP17H01777.

\section{References}

1. Ministry of Health, Labor and Welfare: Measures for Mental Health Depression , http://www.mhlw.go.jp/kokoro/nation/dyp.html

2. Y. Kimura: "Developmental Disabilities" as Medicalization: Focusing the Process of Interpretation in the Educational Setting. The Journal of Educational Sociology, vol. 79, pp. 5-24. (2006)

3. K. Miyagi, S. Kawano, H. Ishii, H. Shimoda: Improvement and Evaluation of Intellectual Productivity Model Based on Work State Transition. 2012 IEEE International Conference on Systems, Man, and Cybernetics, pp. 1491-1496. (2012)

4. H. Shimoda, K. Ooishi, K. Miyagi, K. Uchiyama, H. Ishii, F. Obayashi, M. Iwakawa: An Intellectual Productivity Evaluation Tool Based on Work Concentration. 15th International Conference on Human-Computer Interaction, Vol.16, pp. 364-372. (2013)

5. K. Uchiyama, K. Ooishi, K. Miyagi, H. Ishii, H. Shimoda: Process of Evaluation Index of Intellectual Productivity Based on Work Concentration. Proceedings of ICSTE 2013

6. K. Ueda, S. Shimonaka, H. Shimoda, H. Ishii, F. Obayashi: Quantitative Evaluation of Intellectual Productivity Considering Depth of Concentration. 2017 IEEE International Conference on Systems, Man, and Cybernetics, pp. 758-763. (2017)

7. K. Ueda, H. Shimoda, H. Ishii, F. Obayashi, K. Taniguchi: Development of a New Cognitive Task to Measure Intellectual Concentration Affected by Room Environment. The Fifth International Conference on Human-Environment System. (2016)

8. A. Yasuda, A. Sato: Development of the behavioral inhibition system/behavioral approach system scales. The Japanese Journal of Psychology, Vol. 73, No. 3, pp. 234-242. (2002)

9. A. Wakabayashi: Guidebook for Autism-Spectrum Quotient Adults' version in Japan. Sankyobo. (2016)

10.T. Yagi: Diagnostic Manual of YG Test -Utilization of Personality Survey in Personnel Management-. Institute for Psychological Testing, Inc. (1989)

11.Y. Nakagawa, I. Daibo: Guidebook for the General Health Questionnaire in Japan (Augmented Version). Nihon Bunka Kagakusha Co., Ltd. (2013)

12.I. Fukunishi: Inspection Guide of Global Scale for Depression. Chiba Test Center Co., Ltd. (2012)

13.Y. Uchida, V. Norasakkunkit: The NEET and Hikikomori Spectrum: Assessing the Risks and Consequences of Becoming Culturally Marginalized. Frontiers in Psychology, Vol.6, No.1117 (2015)

14.F. Pedregosa, G. Varoquaux, et al.: Scikit-learn: Machine Learning in Python. JMLR, Vol.12, pp. 2825-2830. (2011) 